\title{
New insights into autocrine cytokines produced by ischemic cardiomyocytes and ventricular remodeling
}

\author{
YU Xian ${ }^{1,2} \&$ LIAO YuHua ${ }^{1,2 *}$ \\ ${ }^{1}$ Laboratory of Cardiovascular Immunology, Institute of Cardiology, Union Hospital, Tongji Medical College, Huazhong University of Science \\ and Technology, Wuhan 430022, China; \\ ${ }^{2}$ Laboratory of Biological Targeted Therapy of the Ministry of Education, Wuhan 430022, China
}

Received April 7, 2015; accepted April 27, 2015; published online July 24, 2015

Citation: Yu X, Liao YH. New insights into autocrine cytokines produced by ischemic cardiomyocytes and ventricular remodeling. Sci China Life Sci, 2015, 58: 915-917, doi: 10.1007/s11427-015-4883-7

Ischemic heart disease is one of the leading causes of death in the world. Although modern therapy of acute myocardial infarction (AMI) can reduce infarct size and result in an increased early survival rate, heart failure after AMI is still a major problem in survivors. Ventricular remodeling is critically important in heart failure after ischemia. It is characterized by changes in ventricular geometry and rigidity, as well as phenotypic modifications and functional changes of cardiomyocytes [1]. Induction of pro-inflammatory cytokines was proved to be associated with the onset and progression of ventricular remodeling [2]. While the heart is not a traditional cytokine-producing organ, cytokines were found locally expressed within the myocardium after AMI in rats [3]. Pro-inflammatory cytokines could activate nuclear factor $\kappa \mathrm{B}(\mathrm{NF}-\kappa \mathrm{B})$, a key transcriptional regulator of inflammation, which further induces cytokine production. This process forms an autocrine amplification loop. Modulation of the cytokines locally expressed in cardiomyocytes per se represents a possible new target for intervention in heart failure after AMI.

Cytokines are produced soon after myocardial ischemic injury, tightly regulate apoptosis of cardiomyocytes, and trigger inflammatory responses. Generally, the main source of cytokines is immunocytes. Tumor necrosis factor- $\alpha$ (TNF- $\alpha$ ), transforming growth factor- $\beta$ (TGF- $\beta$ ) and members of the interleukin (IL) family are secreted by cardio-

*Corresponding author (email: liaoyh27@163.com) myocytes after AMI. Among these, TNF- $\alpha$ is the most widely studied cytokine in cardiovascular disease. It is initially synthesized as a transmembrane protein (mTNF), after cleavage by TNF- $\alpha$-converting enzyme, a free peptide (sTNF) is released into the extracellular space. Both forms have pleiotropic effects through binding to TNF- $\alpha$ receptors (TNFRs). The TNF- $\alpha$ level is elevated in patients with AMI, with the protein evenly distributed between cardiomyocytes and resident cardiac macrophages. This leads to defects in contractility, myocyte apoptosis, and inflammatory signal transduction, as well as extracellular matrix (ECM) synthesis and cardiac regeneration at the chronic stage. We directly verified that TNF- $\alpha$ could be secreted by hypoxic cardiomyocytes in vitro. Hypoxia induces stable expression and translocation of hypoxia inducible factor- $1 \alpha$ (HIF-1 $\alpha$ ), which binds to hypoxia response elements (HREs) in the TNF- $\alpha$ gene $(T N F)$ promoter, resulting in transcription of the gene. The predominant form of TNF- $\alpha$ produced by hypoxic cardiomyocytes is mTNF, which was verified to be released via exosomes. Exosomes containing mTNF transferred it to the targeted cardiomyocytes, induced apoptosis, thus playing an autocrine role (Figure 1) [4]. TNFR1containing exosome-like vesicles were also found in human plasma and modulated TNF-mediated inflammation [5].

TGF- $\beta$ was up-regulated in AMI animal models and progressive coronary artery occlusion. It critically regulates cytokine expression and fibrous tissue deposition, by mediating myofibroblast differentiation and inducing ECM syn- 


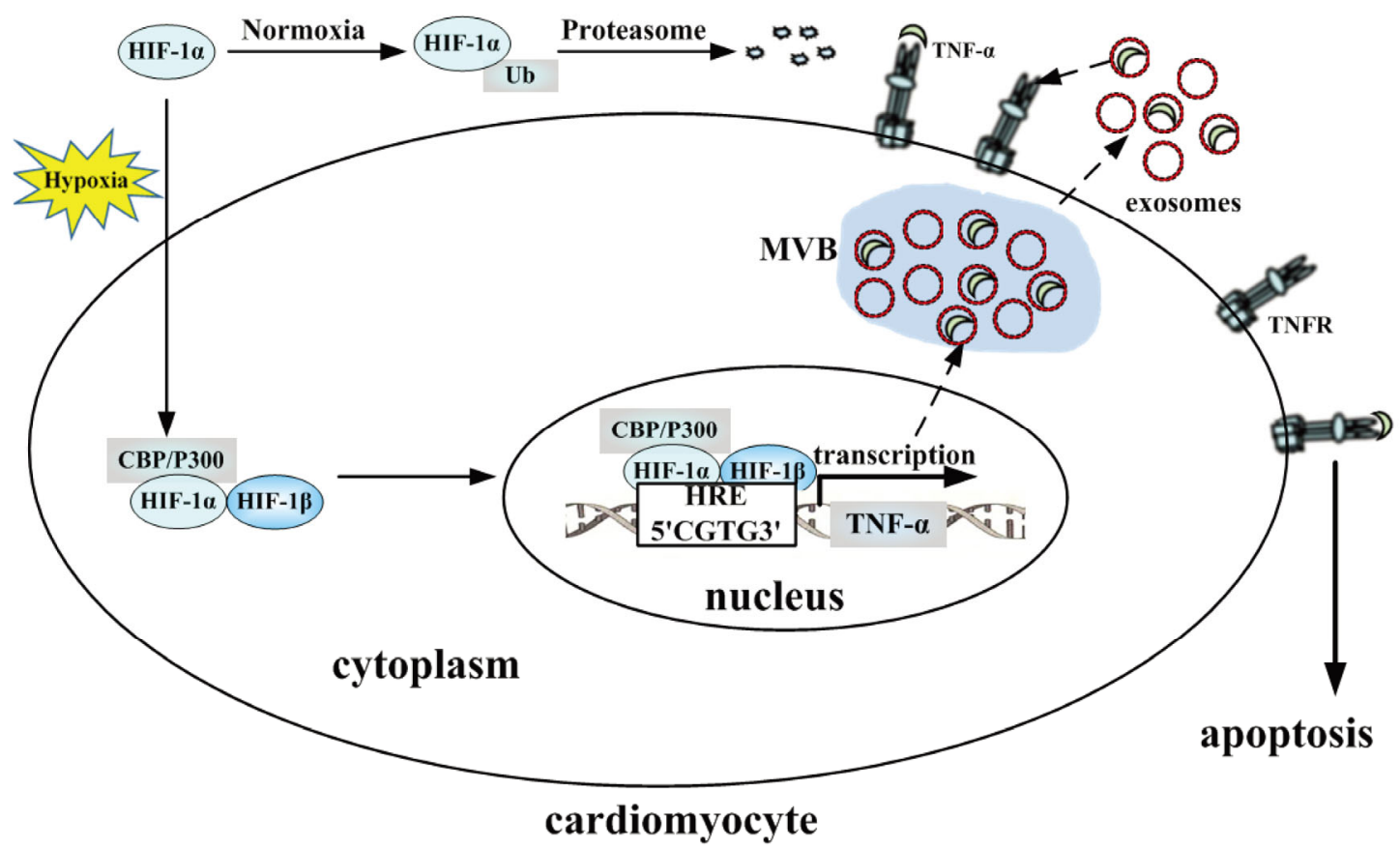

Figure 1 Schematic diagram outlining the autocrine mechanism of tumor necrosis factor- $\alpha$ (TNF- $\alpha$ ) produced by hypoxic cardiomyocytes. CBP/P300: CREB binding protein/EIA binding protein P300 coactivator; HIF: hypoxia inducible factor; HRE: hypoxia response element; MVB: multivesicular body; TNFR: tumor necrosis factor- $\alpha$ receptor; Ub: ubiquitin.

thesis. Among ILs, IL-1 $\beta$, IL-6, IL-10, and IL-33 were verified to be released by the myocardium after ischemic stress. IL-1 $\beta$ and IL- 6 are pro-inflammatory cytokines, which exhibit overlapping characteristics with TNF- $\alpha$. Both these pro-inflammatory cytokines are up-regulated in an AMI model and contribute to ventricular remodeling. IL-10 and IL-33 are potent anti-inflammatory cytokines, which inhibit macrophage infiltration and the production of proinflammatory cytokines, ultimately contributing to improved ventricular function.

The exact autocrine mechanism of cytokines in cardiomyocytes involves stressors triggered by ischemia, activation of related signal transduction pathways, critical transcription factors, and the secretory pathway. Ischemia and hypoxia are direct triggers for cytokine production in AMI. They can activate the neuro-endocrine-immune system, leading to renin-angiotensin-aldosterone system activation, an excited sympathetic nervous system, and inflammatory cytokine synthesis.

Mitogen-activated protein kinase (MAPK) subfamilies constitute the common pathway after neuro-endocrineimmune system activation. Three major MAPK subfamilies found in the heart include extracellular signal-regulated kinases (ERKs), c-Jun N-terminal kinases (JNKs), and p38 MAPKs. Activation of p38 MAPKs is sufficient to induce IL-1, IL-6, and TNF- $\alpha$ expression in cardiomyocytes, and contributes to ventricular remodeling. JNK and ERK signaling pathways are activated after ischemia; whether they can induce cytokine production, needs further investigation.
The receptor activator of NF-kB ligand (RANKL) signaling pathway is another important pathway that was found to be activated in ischemic heart tissue and specifically induce TNF- $\alpha$, IL- $1 \alpha$, and IL-1 $\beta$ expression during myocardial remodeling [6].

Transcriptional regulation occurs via specific interaction of nuclear transacting protein factors with corresponding cis-elements in the gene promoter. HIF-1 is the master factor and direct regulator of cellular adaption to hypoxic stress. It is a hetero-dimeric protein complex composed of HIF- $1 \alpha$ and HIF-1 $\beta$. HIF- $1 \alpha$ is degraded through the VHL (von Vippel-Lindau)-dependent ubiquitin-proteasome pathway under normoxia. This pathway is blocked under hypoxia. Accumulated HIF- $1 \alpha$ dimerizes with HIF- $1 \beta$, forming HIF, which induces transcription via interaction with HREs containing a core 5 '-CGTG- 3 ' motif in the regulatory regions of the target genes. In recent studies, HIF- $1 \alpha$ was found to take part in cytokine transcriptional regulation. Both the $I L 1 B$ and $I L 20$ genes carry HREs in their promoter regions that were verified to be functional. In our study, HIF-1 $\alpha$ was activated and stably expressed in primary cultured cardiomyocytes under hypoxia, bound the HRE between nucleotides -1295 and -1292 in the 5 -flanking region of the $T N F-\alpha$ promoter as the functional cis-acting element required for hypoxic activation, and eventually induced $T N F$ gene transcription and protein biogenesis [4]. NF- $\mathrm{KB}$ is another transcription factor responsible for TNF- $\alpha$ expression. It is comprised of two subunits and retained in the cytoplasm by interaction with the protein inhibitor of $\kappa \mathrm{B}$ (I $\mathrm{KB}$ ). 
I $\kappa \mathrm{B}$ is phosphorylated after NF- $\kappa \mathrm{B}$ activation which leads to $\mathrm{I} \kappa \mathrm{B}$ degradation and $\mathrm{NF}-\kappa \mathrm{B}$ translocation.

Cytokine secretion is a complex process that is highly regulated via different pathways. The majority of cytokines are processed and transported through the classical pathway. In this pathway, cytokines are synthesized in the endoplasmic reticulum (ER) and trafficked to the Golgi complex for further processing. In the trans-Golgi network, they are loaded into vesicles for constitutive delivery to the cell surface or transit via recycling endosomes. In a non-classical secretory pathway, cytokines move directly from their point of synthesis in the cytoplasm to the external milieu. Different mechanisms have been proposed regarding the passage through the plasma membrane, including the use of membrane transporters, exosome release from multi-vesicular bodies, and micro-vesicle shedding from the plasma membrane. The exact mechanism is often specific to a unique cytokine and cell type. TNF- $\alpha$ secretion by macrophages occurs via the classical secretory pathway. Macrophages also secrete IL- 6 and IL-10 by the classical pathway by recycling endosomes [7]. Cytokines such as IL-1 $\beta$, lacking the $\mathrm{N}$-terminal signal sequence required for ER entry, are synthesized in the cytoplasm, and thereafter released from cells by non-classical pathways [8]. Our group [4] found that TNF- $\alpha$ was released through exosomes from hypoxic cardiomyocytes. Exosomes are nano-vesicles released from the cells via exocytosis; they have been shown to carry and transfer a wide range of cargos, including proteins, lipids, and nucleic acids, and mediate proximal as well as distal cell-to-cell signaling.

During heart failure after AMI, ischemic stimulation activates HIF-1 $\alpha$ and the neuro-endocrine-immune system. Further activation of NF- $\kappa \mathrm{B}$ induces transcription of cyto- kines, which are trafficked to the targets cells through different pathways. Accumulating evidence has proved that cytokines are synthesized by ischemic cardiomyocytes, which provides a new insight into the inflammatory processes involved in ventricular remodeling. Increase in published studies on this topic makes exosomes (the newly reported carriers in cytokine transfer) the possible and potential targets for immune modulation in future.

This work was supported by the National Basic Research Program of China (2007CB512000, 2007CB512005).

1 Heusch G, Libby P, Gersh B, Yellon D, Böhm M, Lopaschuk G, Opie L. Cardiovascular remodelling in coronary artery disease and heart failure. Lancet, 2014, 383: 1933-1943

2 Mann DL. Innate immunity and the failing heart: the cytokine hypothesis revisited. Circ Res, 2015, 116: 1254-1268

3 Seropian IM, Toldo S, Van Tassell BW, Abbate A. Anti-inflammatory strategies for ventricular remodeling following ST-segment elevation acute myocardial infarction. J Am Coll Cardiol, 2014, 63: 1593-1603

4 Yu X, Deng L, Wang D, Li N, Chen X, Cheng X, Yuan J, Gao X, Liao M, Wang M, Liao Y. Mechanism of TNF- $\alpha$ autocrine effects in hypoxic cardiomyocytes: initiated by hypoxia inducible factor $1 \alpha$, presented by exosomes. J Mol Cell Cardiol, 2012, 53: 848-857

5 Zhang J, Hawari FI, Shamburek RD, Adamik B, Kaler M, Islam A, Liao DW, Rouhani FN, Ingham M, Levine SJ. Circulating TNFR1 exosome-like vesicles partition with the LDL fraction of human plasma. Biochem Biophys Res Commun, 2008, 366: 579-584

6 Ock S, Ahn J, Lee SH, Park H, Son JW, Oh JG, Yang DK, Lee WS, Kim HS, Rho J, Oh GT, Abel ED, Park WJ, Min JK, Kim J. Receptor activator of nuclear factor- $\mathrm{KB}$ ligand is a novel inducer of myocardial inflammation. Cardiovasc Res, 2012, 94: 105-114

7 Murray RZ, Stow JL. Cytokine secretion in macrophages: SNAREs, Rabs, and membrane trafficking. Front Immunol, 2014, 5: 538

8 Stow JL, Murray RZ. Intracellular trafficking and secretion of inflammatory cytokines. Cytokine Growth Factor Rev, 2013, 24: 227-239

Open Access This article is distributed under the terms of the Creative Commons Attribution License which permits any use, distribution, and reproduction in any medium, provided the original author(s) and source are credited. 